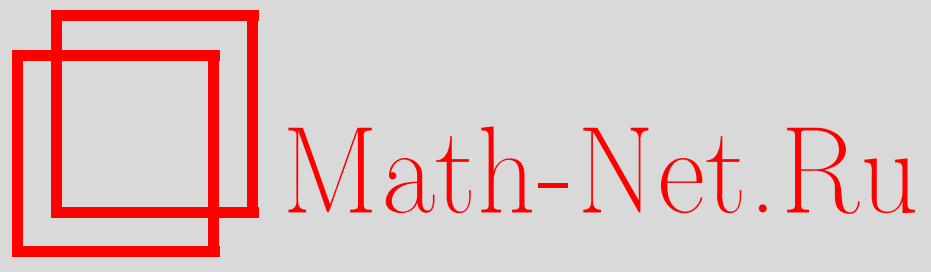

А. Я. Белов, О многообразиях, порожденных кольцом, конечномерным над центроидом, УМН, 2007, том 62, выпуск 2, 171-172

DOI: https://doi.org/10.4213/rm6805

Использование Общероссийского математического портала Math-Net.Ru подразумевает, что вы прочитали и согласны с пользовательским соглашением http://www . mathnet.ru/rus/agreement

Параметры загрузки:

IP : 54.164 .48 .24

26 апреля 2023 г., 13:13:18

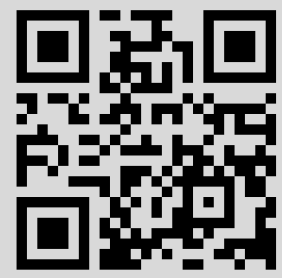




\section{О многообразиях, порожденных кольцом, конечномерным над центроидом}

\section{А. Я. Белов}

Известно, что многообразия ассоциативных алгебр над бесконечным полем локально порождаются конечномерной алгеброй [1]. Тем не менее уже для алгебр над конечным полем это неверно. Простейшим примером служит многообразие, порожденное полупрямым произведением конечного поля $k$ и кольца многочленов $k[x]$. Более интересный пример - многообразие, порожденное матрицами вида $\left(\begin{array}{cc}P & * \\ 0 & P^{q}\end{array}\right)$, где $P \in k[x]$, поле $k$ удовлетворяет тождеству $z^{q}=z$. Тем не менее любое многообразие ассоциативных алгебр над произвольным ассоциативно-коммутативным кольцом $\Phi$ порождается подалгеброй алгебры, конечномерной над центром [2]. Основная теорема данной работы такова:

ТЕОрема 1. Идеал тождеств многообразия М алгебр произвольной сигнатуры, которое порождается алгеброй, конечномерной над центроидом, есть пересечение идеалов тождеств конечной алгебры и однородного многообразия.

Если многообразие $\mathfrak{M}$ унитарно замкнуто, то оно порождается алгеброй, конечномерной над иентром.

ОПРеДЕЛЕНиЕ 1. Многообразие $\mathfrak{M}$ называется однородным, если каждое тождество выполняется в $\mathfrak{M}$ вместе со всеми своими компонентами, однородными по каждой переменной.

Однородные многообразия - это в точности те многообразия, которые устойчивы относительно центральных расширений:

ПреДЛОЖЕНИЕ 1. Пустъ $\mathfrak{M}$ - однородное многообразие алгебр, не обязательно ассоциативных. $B \in \mathfrak{M}, C$ - ассочиативно-коммутативное колъцо. Тогда алгебра $B \otimes C$ принадлежит $\mathfrak{M}$.

Для многообразий, порожденных конечно порожденными ассоциативными кольцами, справедливо и обратное утверждение.

Мы рассматриваем кольца и алгебры над нётеровым ассоциативно-коммутативным кольцом $\Phi$. Поскольку $n$-кратное сложение любого элемента с самим собой определено, можно считать, что $\Phi$ содержит 1 и, следовательно, содержит $\mathbb{Z}_{n}$.

Назовем модулем существенного кручения подмодуль $\operatorname{Tor}_{\mathrm{Es}}(M) \subseteq M$, состоящий из элементов, аннуляторы которых не содержатся в радикале Джекобсона $J(R)$. Через $\operatorname{Tor}_{\Phi}(M)$ будем обозначать модуль кручения для кольца $\Phi$ (подмодуль в $M$ ).

ПредЛОЖение 2. а) Пусть $M$ - нётеров модуль над первичным нётеровым кольцом $R$, $\operatorname{Tor}(M)$ - подмодуль кручения. Тогда $M$ вкладывается в прямую сумму своих факторов $M / I \cdot M \oplus M / \operatorname{Tor}(M)$ для некоторого собственного идеала $I$ кольща $R$.

б) Если при этом $R=\Phi \otimes R_{0}$ есть тензорное произведение нётеровых первичных колец, с единицей, то $M$ вкладывается в прямую сумму своих факторов $M / I \cdot M \oplus$ $M / \operatorname{Tor}_{\Phi}(M)$ для некоторого собственного идеала I кольца $\Phi$.

в) В условиях $n$. а) пусть $R$ - нётерово кольио без идеалов с нулевым пересечением, так что его фактор по радикалу Джекобсона $\bar{R}=R / J(R)$ первичен. Тогда $M$ вкладивается в прямую сумму своих факторов $M / I \cdot M \oplus M / \operatorname{Tor}_{\text {Ess }}(M)$ для некоторого собственного идеала I кольца $R$.

г) В условиях п. а) пусть $R$ - произвольное нётерово кольцо, $\bar{R}_{i}$ - первичнъе кольца и $R / J(R)=\bigoplus \bar{R}_{i}, R_{i}=\pi^{-1}\left(\bar{R}_{i}\right)$. Тогда $M$ вкладывается в прямую сумму своих факторов $M / R_{i}^{k} M$ для некоторого натурального $k$.

ЛЕмма 1. Пусть для некоторых элементов $e_{i}$ из $\Phi$-модуля $M$ и некоторых $\lambda_{i j} \in \Phi$ выполняется система уравнений $\sum_{i=1}^{n} \lambda_{i j} e_{i}=0, j=1, \ldots, n$.

Тогда при всех $i$ верно, что $e_{i} \Delta=0$, где $\Delta=\operatorname{det}\left(\left(\lambda_{i j}\right)\right)$. 
ДокАЗАтЕльство. Пусть $M_{i j}$ - минор матрицы $\Lambda=\left(\lambda_{i j}\right), M=\left(M_{i j}\right)$. Тогда $\Lambda M=\Delta E$, или $\sum_{j} M_{i j} \lambda_{j k}=\Delta \delta_{i k}\left(\delta_{i k}=0\right.$ при $i \neq k$ и $\delta_{i k}=1$ при $i=k$.) Поэтому $0=\sum_{j k} M_{i j} \lambda_{j k} e_{k}=\Delta e_{i}$.

ПреДЛОжениЕ 3. а) Однородная компонента тождества $f$ по переменной $x$ обращается в нуль при умножении на любое значение определителя Вандермонда, составленного из любых элементов кольца $\Phi$ и имеющего порлдок, равный степени тождества $f$ nо $x$.

б) Пусть кольцо $\bar{\Phi}$ бесконечно. Тогда полиоднородные (т.е. однородные по каждой переменной) компоненты тождеств из Ф-алгебры А имеют существенное кручение.

в) Пусть кольцо $\Phi$ для любого $n$ содержит обратный элемент к некоторому значению определителя Вандермонда порядка п. Тогда любое тождество равносилъно набору своих полиоднородных компонент и любое многообразие $\Phi$-алгебр однородно.

ДокАзАтельство. Пункты б) и в) следуют из п. а). Докажем п. а).

Пусть $f=\sum_{i=0}^{n} f_{i}, f_{i}$ - компонента $f$ степени однородности $i$ относительно переменной $x$. Результат подстановки $\lambda^{k} x \rightarrow x$ в $f$ есть тождество $\sum_{i=0}^{n} \lambda^{k i} f_{i}$, являющееся следствием тождества $f$. Поэтому п. а) следует из леммы 1.

Лемма 2. Пусть кольио $\Phi$ содержит несколько идеалов $I_{\alpha} \ni E_{\alpha}$, где $E_{\alpha}-$ семейство ортогональных идемпотентов, в сумме дающих единицу. Тогда любой $\Phi$-модуль (любая $\Phi$-алгебра) разлагается в прямую сумму $\Phi / I_{\alpha}$-модулей (алгебр), являющихся его факторами.

ПредложениЕ 4. Любал конечномерная $R$-алгебра над бесконечным кольцом $R$, не имеющая $R$-кручения (тем самым $R$ - первичное кольцо), порождает однородное многообразие.

ДокАЗАтЕльСтво теоремы 1. Пусть $A$ - алгебра, вкладывающаяся в конечномерную $R$-алгебру. Воспользовавшись предложением 2 , мы можем считать алгебру $A$ прямой суммой $R_{i}$-алгебр $A_{i}$, причем каждая $A_{i}$ не имеет $R_{i}$-кручения. В силу предложения 4 при бесконечных $R_{i}$ алгебра $A_{i}$ порождает однородное многообразие, и теорема 1 доказана.

Пусть $A \subseteq \widetilde{A}, \widetilde{A}$ - алгебра, вкладывающаяся в конечномерную $R$-алгебру. Тогда если многообразие $\operatorname{Var}(A)$ однородно, то центральное расширение $A[R]$ подалгебры $A$ конечномерно как $R$-алгебра и порождает то же многообразие, что и $A$. Таким образом, из локальной представимости ассоциативных относительно свободных колец [2] вытекает

Теорема 2. Пусть в каждом подмногообразии $\mathfrak{M}$ произвольной сигнатуры относительно свободная алгебра представима (т.е. вкладывается в конечномерную над центром). Тогда любое однородное подмногообразие $\mathfrak{M}$ локально порождается алгеброй, конечномерной над центроидом.

СледСтвиЕ 1. Пусть фактор по любому максимальному идеалу кольца Ф бесконечен. Тогда любое многообразие Ф-алгебр однородно и локально порождается алгебрами, конечномерными над иентроидом.

\section{Список литературы}

[1] А. Р. Кемер, “Тождества конечно порожденных алгебр над бесконечным полем", Изв. АН СССР. Сер. матем., 54:4 (1990), 726-753. [2] А. Я. Белов, Дисс. .. докт. физ.-матем. наук, МГУ, М., 2002.

\section{А. Я. Белов (А. Yа. Belov)}

Московский институт открытого образования;

Hebrew University of Jerusalem

E-mail: kanel@mccme.ru
Представлено А. В. Михалёвым Принято редколлегией 12.03.2007 